\title{
SHIFT INVARIANT MEASURES AND SIMPLE SPECTRUM
}

BY

A. KŁOPOTOWSKI (PARIS) AND M. G. NADKARNI (MUMBAI)

In memoriam: Anzelm Iwanik

\begin{abstract}
We consider some descriptive properties of supports of shift invariant measures on $\mathbb{C}^{\mathbb{Z}}$ under the assumption that the closed linear span (in $L^{2}$ ) of the co-ordinate functions on $\mathbb{C}^{\mathbb{Z}}$ is all of $L^{2}$.
\end{abstract}

Introduction. Let $\Omega=\mathbb{C}^{\mathbb{Z}}$ be equipped with the usual product $\sigma$ algebra and a probability measure $\mu$ invariant under the shift

$$
T\left(\omega_{n}\right)_{n=-\infty}^{\infty}=\left(y_{n}\right)_{n=-\infty}^{\infty}, \quad y_{n}=\omega_{n+1}, \quad n \in \mathbb{N} .
$$

Assume that the projection maps $X_{n}(\omega)=\omega_{n}, n \in \mathbb{Z}$, are in $L^{2}(\Omega, \mu)$ and that their closed linear span is $L^{2}(\Omega, \mu)$. This is equivalent to saying that the unitary operator $U_{T} f=f \circ T, f \in L^{2}(\Omega, \mu)$, has multiplicity one. The purpose of this note is to discuss some properties of $\mu$ (with regard to its support) and to draw some conclusions about the transformation $T$. It is a continuation of the theme discussed in [1] and [2].

\section{Formulation of the problem}

1.1. Let $\mathbb{Z}$ be divided into two disjoint sets $A$ and $B$; let $\Omega_{A}=\mathbb{C}^{A}$, $\Omega_{B}=\mathbb{C}^{B}$ and we identify $\Omega$ with $\Omega_{A} \times \Omega_{B}$ in the usual fashion. Let $\Pi_{A}$ and $\Pi_{B}$ denote the projection maps of $\Omega$ to $\Omega_{A}$ and $\Omega_{B}$ respectively and let $\mu_{A}=\mu \circ \Pi_{A}^{-1}, \mu_{B}=\mu \circ \Pi_{B}^{-1}$ be the marginal measures. Since $U_{T}$ has simple spectrum, each $f \in L^{2}(\Omega, \mu)$ is the $L^{2}$ limit of a sequence $f_{n}, n=1,2, \ldots$, where $f_{n}$ 's are finite linear combinations of $X_{k}, k \in \mathbb{Z}$. If $f_{n}=\sum a_{n, k} X_{k}$ is such a finite sum, then

$$
f_{n}=u_{n}+v_{n},
$$

2000 Mathematics Subject Classification: Primary 60A05, 47A35; Secondary 28D05, $37 \mathrm{Axx}$.

Key words and phrases: shift, simple spectrum, good set, linked component, good measure. 
where $u_{n}$ is the sum of $a_{n, k} X_{k}$ for $k \in A$ and $v_{n}$ is the sum of $a_{n, k} X_{k}$ for $k \in B$. Since $f_{n} \rightarrow f$ in $L^{2}$, it is natural to ask if $f=u+v \mu$-a.e., where $u$ and $v$ are Borel functions on $\Omega_{A}$ and $\Omega_{B}$ respectively, and if so, whether this sheds some light on the structure of $\mu$.

2. Set-theoretic version. We will first formulate and answer a simpler set-theoretic version of the questions of the previous section. At the descriptive set-theoretic level we have an interesting example and some questions. (See Section 3.)

Let $X$ and $Y$ be two non-empty sets (these correspond to $\Omega_{A}$ and $\Omega_{B}$ of the previous section). Let $S$ be a non-empty subset of $X \times Y$.

2.1. Definition. We say that $S$ is good if every complex-valued function $f$ on $S$ can be expressed in the form

$$
f(x, y)=u(x)+v(y), \quad(x, y) \in S,
$$

where $u$ and $v$ are functions on $X$ and $Y$ respectively.

2.2. Definition. We say that $S$ is sequentially good if every complexvalued function $f$ on $S$ is the limit

$$
f(x, y)=\lim _{n \rightarrow \infty}\left(u_{n}(x)+v_{n}(y)\right), \quad(x, y) \in S,
$$

where $u_{n}, v_{n}, n=1,2, \ldots$, are functions on $X$ and $Y$ respectively.

We will see that a set is good if and only if it is sequentially good (see 2.11)

2.3. Example. Let $X=Y=\mathbb{Z}$. The set

$$
S=\{(n, n-1): n \in \mathbb{Z}\} \cup\{(n, n): n \in \mathbb{Z}\} \subseteq \mathbb{Z} \times \mathbb{Z}
$$

is good. To see this, fix any complex-valued function $f$ on $S$. If we put $u(0)=$ $c$, where $c$ is an arbitrary constant, then $v(0)=f(0,0)-c$. Having defined $v(0)$, we see that $u(1)=f(1,0)-v(0), v(1)=f(1,1)-u(1)$. Proceeding thus we see that $u$ and $v$ are uniquely defined as soon as we fix the value of $u(0)$.

This gives a method to describe good subsets of $X \times Y$.

2.4. Definition. Two arbitrary points $(x, y),(z, w) \in S \subseteq X \times Y$ ( $S$ is not necessarily good) are said to be linked (and we write $(x, y) L(z, w)$ ) if there exists a finite sequence of points $\left\{\left(x_{1}, y_{1}\right),\left(x_{2}, y_{2}\right), \ldots,\left(x_{n}, y_{n}\right)\right\}$ in $S$ (called a link of length $n$ joining $(x, y)$ to $(z, w)$ ) such that:

(i) $\left(x_{1}, y_{1}\right)=(x, y),\left(x_{n}, y_{n}\right)=(z, w)$;

(ii) for any $1 \leq i \leq n-1$ exactly one of the following equalities holds:

$$
x_{i}=x_{i+1}, \quad y_{i}=y_{i+1}
$$


(iii) for any $i, 1 \leq i \leq n-2$, it is not possible to have $x_{i}=x_{i+1}=x_{i+2}$ or $y_{i}=y_{i+1}=y_{i+2}$.

One can verify easily that $L$ is an equivalence relation.

2.5. Definition. An equivalence class of $L$ is called a linked component of $S$. If $(x, y) \in S$, then the equivalence class to which $(x, y)$ belongs is called the linked component of $(x, y)$. Two points $(x, y),(z, w) \in S$ are said to be uniquely linked if there is a unique link joining $(x, y)$ to $(z, w)$.

REMARK. Note that linked components of $S$ are singletons if and only if $S$ is the graph of a one-to-one function.

It is easy to verify

2.6. Proposition. Let $Q$ be a linked component of $S$. Then the following properties are equivalent:

(i) any two points of $Q$ are uniquely linked;

(ii) some two points of $Q$ are uniquely linked;

(iii) for some $(x, y) \in Q$ the singleton $\{(x, y)\}$ is the only link joining $(x, y)$ to itself.

2.7. Definition. A linked component of $S \subseteq X \times Y$ is said to be uniquely linked if any two points in it are uniquely linked.

2.8. Definition. By a trivial link joining $(x, y)$ to itself we mean the link consisting of the singleton $\{(x, y)\}$. A non-trivial link joining $(x, y)$ to itself is called a loop.

Proposition 2.6 says that a linked component is uniquely linked if it has no loops.

In [1] we have proved:

2.9. Theorem. A linked subset $S \subseteq X \times Y$ is good if and only if it is uniquely linked. More generally, a subset $S \subset X \times Y$ is good if and only if each of its linked components is uniquely linked.

2.10. Remark. Assume that a subset $S \subset X \times Y$ is linked, but not uniquely linked. Then $S$ admits a loop

$$
L=\left\{\left(x_{1}, y_{1}\right),\left(x_{2}, y_{2}\right), \ldots,\left(x_{n}, y_{n}\right)\right\}, \quad\left(x_{1}, y_{1}\right)=\left(x_{n}, y_{n}\right),
$$

which we can assume to be of shortest length. Then either $x_{1}=x_{2} \neq x_{n-1}$ or $y_{1}=y_{2} \neq y_{n-1}$. Let $f$ be a function on $L$ with the property that $f(x, y)=$ $u(x)+v(y),(x, y) \in L$, for some functions $u$ and $v$ on $X$ and $Y$ respectively. Then, on the loop $L, f$ satisfies

$$
f\left(x_{1}, y_{1}\right)-f\left(x_{2}, y_{2}\right)+\ldots-f\left(x_{n-1}, y_{n-1}\right)=0 .
$$

Any function on $L$ not satisfying the above equality therefore cannot be written in the form $u(x)+v(y),(x, y) \in L$. 
2.11. TheOrem. $S$ is sequentially good if and only if $S$ is good.

Proof. If $S$ is good, then clearly $S$ is sequentially good. Assume now that $S$ is sequentially good but not good. Then $S$ admits a loop $L=$ $\left\{\left(x_{1}, y_{1}\right),\left(x_{2}, y_{2}\right), \ldots,\left(x_{n}, y_{n}\right)\right\},\left(x_{1}, y_{1}\right)=\left(x_{n}, y_{n}\right)$, of shortest length and we can define a function $f$ on $L$ which satisfies

$$
f\left(x_{1}, y_{1}\right)-f\left(x_{2}, y_{2}\right)+\ldots-f\left(x_{n-1}, y_{n-1}\right) \neq 0 .
$$

Clearly for $\varepsilon>0$ sufficiently small, any $g$ on $L$ which satisfies

$$
\left|f\left(x_{i}, y_{i}\right)-g\left(x_{i}, y_{i}\right)\right|<\varepsilon, \quad 1 \leq i \leq n-1,
$$

will also satisfy

$$
g\left(x_{1}, y_{1}\right)-g\left(x_{2}, y_{2}\right)+\ldots-g\left(x_{n-1}, y_{n-1}\right) \neq 0,
$$

and so cannot be expressed in the form $g(x, y)=u(x)+v(y)$, whence the function $f$ cannot be a pointwise limit of a sequence of functions of the form $g_{n}(x, y)=u_{n}(x)+v_{n}(y)$. So $L$, hence $S$, is not sequentially good and the theorem follows.

2.12. TheOREM. If a subset $S \subseteq X \times Y$ is uniquely linked, then $S$ is of the form $G \cup H$, where $G$ is the graph of a function $g$ on a subset of $X$ and $H$ is the graph of a function $h$ on a subset of $Y$. More generally, if each linked component of $S$ is uniquely linked, then also $S$ is a union of two graphs $G$ and $H$ as above.

Proof. This result can be verified for the case when $S$ is as in Example 2.3, and essentially the same method allows us to prove the result generally for any uniquely linked $S$. Indeed, let $S$ be uniquely linked. Fix $(a, b) \in S$ and let:

$$
\begin{gathered}
G_{1}(a, b)=\{(x, y):(x, y) \text { is linked to }(a, b) \text { by a path of even length } \\
\text { with the first step in the vertical direction }\}, \\
G_{2}(a, b)=\{(x, y):(x, y) \text { is linked to }(a, b) \text { by a path of odd length } \\
\text { with the first step in the horizontal direction }\}, \\
H_{1}(a, b)=\{(x, y):(x, y) \text { is linked to }(a, b) \text { by a path of even length } \\
\text { with the first step in the horizontal direction }\}, \\
H_{2}(a, b)=\{(x, y):(x, y) \text { is linked to }(a, b) \text { by a path of odd length } \\
\text { with the first step in the vertical direction }\}, \\
G=G(a, b)=G_{1}(a, b) \cup G_{2}(a, b), \quad H=H(a, b)=H_{1}(a, b) \cup H_{2}(a, b) .
\end{gathered}
$$

It is easy to see that $G$ and $H$ are the graphs of functions defined on subsets of $X$ and $Y$ respectively and that $G \cup H=S$. (We also note that $G \cap H=\{(a, b)\}$. Further if $(c, d)$ is any other point in $S$, then $G(a, b)=$ 
$G(c, d)$ if $(c, d) \in G(a, b)$ while if $(c, d) \notin G(a, b)$, then $G(a, b) \triangle G(c, d)=$ $\{(a, b),(c, d)\}$. Thus, in any case, $G(a, b)$ differs from $G(c, d)$ by at most two points.)

To prove the second part, let $S$ be good and let $\mathcal{P}$ denote the partition of $S$ into uniquely linked components. Let $C$ be a cross-section of $\mathcal{P}$, by which we mean a subset of $S$ which meets every set in $\mathcal{P}$ in exactly one point. If $(a, b)$ and $(c, d)$ are distinct points of $C$, then $G(a, b)$ and $G(c, d)$ have disjoint projections on $X$, while $H(a, b)$ and $H(c, d)$ have disjoint projections on $Y$. (Here $G(a, b)$ etc. are taken with respect to the linked component to which $(a, b)$ belongs.) So the sets $G=\bigcup_{(a, b) \in C} G(a, b), H=\bigcup_{(a, b) \in C} H(a, b)$ are graphs of the required kind, with their union equal to $S$. The theorem is proved.

Note that the way $G$ and $H$ are obtained, we have in addition $G \cap H=C$, a cross-section of $\mathcal{P}$.

\section{Descriptive set-theoretic version}

3.1. Assume now that $X=Y=[0,1]$, equipped with the usual Borel structure, and that $S \subset X \times Y$ is a Borel set such that every Borel function $f$ on $S$ is of the form $f(x, y)=u(x)+v(y)$. Then $S$ is good, for otherwise $S$ will admit a loop $L$ (a finite set, hence a Borel set) and we can define a Borel function on $S$ which cannot be written in the form $u+v$ on the loop $L$, with $u, v$ defined on $X$ and $Y$ respectively.

It can be shown that even if $f$ is a Borel function on a good Borel set $S$, the functions $u$ and $v$ need not be Borel. However, if the decomposition into linked components of a good Borel set $S$ admits a Borel cross-section, then $u$ and $v$ can be chosen to be Borel for every Borel $f$ on $S$.

3.2. Let $(a, b) \in S \subseteq X \times Y$. The linked component of $(a, b)$ is obtained as a union $\bigcup_{n=1}^{\infty} Q_{n}$, where

$$
\begin{array}{ll}
Q_{1}=(X \times\{b\}) \cap S, & P_{1}=\Pi_{1} Q_{1}, \\
Q_{2}=\left(\Pi_{1}^{-1} P_{1}\right) \cap S, & P_{2}=\Pi_{2} Q_{2}, \\
Q_{3}=\left(\Pi_{2}^{-1} P_{2}\right) \cap S, & P_{3}=\Pi_{1} Q_{3},
\end{array}
$$

and so on. If $n$ is odd, we have

$$
\begin{aligned}
P_{n} & =\Pi_{1} Q_{n}, & Q_{n+1} & =\left(\Pi_{1}^{-1} P_{n}\right) \cap S, \\
P_{n+1} & =\Pi_{2} Q_{n+1}, & Q_{n+2} & =\left(\Pi_{2}^{-1} P_{n+1}\right) \cap S, \ldots
\end{aligned}
$$

Hence, if $S \subseteq X \times Y$ is a Borel set, then each linked component of $S$ is a countable union of analytic sets, hence the equivalence relation $L$ decomposes $S$ into at least analytic sets. We do not know if a linked component of 
a Borel set is a Borel set. However, a uniquely linked component of a Borel set is Borel.

The quotient Borel structure on the partition $\mathcal{P}$ of linked components need not be standard Borel; indeed, it can be like the quotient Borel structure on the orbit space of an ergodic automorphism, as the following example shows.

3.3. Example (due to Rana Barua and S. M. Srivastava). Let $X=Y=$ $[0,1]$ and let $0<\theta<1$ be an irrational number. Let $J, M, N$ respectively be the line segments in $[0,1] \times[0,1]$ joining $(\theta, 0)$ to $(1,1-\theta),(0,0)$ to $(1,1)$, $(0,1-\theta)$ to $(\theta, 1)$, and let $S$ be the union of $J, M, N$. It is easy to see that the linked components of $S$ are countable and they induce a partition on $M$ consisting of orbits of the map

$$
K:(x, x) \mapsto(x+\theta(\bmod 1), x+\theta(\bmod 1)) .
$$

Since $\theta$ is irrational, this is the Vitali partition of $M$, so the quotient Borel structure on the partition of $S$ into its linked (actually uniquely linked) components is not standard Borel.

This example is indeed a variant of an example due to M. Laczkovich [3] discovered to answer some other question in descriptive set theory.

It can be shown that if $A$ is the line segment joining $(0,0)$ to $(1,1)$, then $1_{A}$ cannot be written as a sum of Borel measurable $u$ and $v$.

3.14. It is not known if the graphs $G$ and $H$ of Theorem 2.12 can be chosen to be Borel when $S$ is good and Borel. The answer is affirmative if the partition of $S$ into uniquely linked components admits a Borel cross-section.

\section{Measure-theoretic version}

4.1. Definition. Let $m$ be a probability measure on Borel subsets of $X \times Y(X=Y=[0,1])$. Call $m$ good if every function $f \in L^{2}(X \times Y, m)$ is of the form $f=u+v$ m-a.e. with $u \in L^{2}\left(X, m_{X}\right), v \in L^{2}\left(Y, m_{Y}\right)$, where $m_{X}, m_{Y}$ are the marginals of $m$ on $X$ and $Y$ respectively.

4.2. Definition. We say that $m$ is sequentially good if every function $f \in L^{2}(X \times Y, m)$ is the $L^{2}$ limit of a sequence $f_{n}, n=1,2, \ldots$, of functions of the form

$$
f_{n}=u_{n}+v_{n}, \quad u_{n} \in L^{2}\left(X, m_{X}\right), \quad v_{n} \in L^{2}\left(Y, m_{Y}\right) .
$$

Clearly, if $m$ is good, then $m$ is sequentially good. It is not known if the converse holds, even in a weaker sense, i.e., whether, when $m$ is sequentially good, every $f \in L^{2}(X \times Y, m)$ is of the form $f=u+v m$-a.e., with $u, v$ merely Borel measurable (and not necessarily in $L^{2}$ ). We will show below that if $m$ is sequentially good, then $m$ admits a Borel support $S$ (not necessarily a closed set) which is good, so that when $m$ is sequentially good, every 
$f \in L^{2}(X \times Y, m)$ is of the form $f=u+v m$-a.e. It is not claimed that $u$ and $v$ can be chosen to be Borel, but they can be so chosen if the partition of $S$ into uniquely linked components admits a Borel cross-section.

4.3. Definition. Say that a subset $S$ of $X \times Y$ is sequentially good for a collection $\mathcal{F}$ of functions on $S$ if every $f \in \mathcal{F}$ is of the form

$$
f(x, y)=\lim _{n \rightarrow \infty}\left(u_{n}(x)+v_{n}(y)\right), \quad(x, y) \in S,
$$

with $u_{n}$ and $v_{n}$ functions on $X$ and $Y$ respectively.

Assume now that $S$ is sequentially good for an algebra $\mathcal{F}$ of functions on $S$ which is closed under conjugation and separates points of $S$. Then in fact $S$ is sequentially good (hence good). For otherwise $S$ will admit a loop $L$. The restriction of functions in $\mathcal{F}$ to $L$ (denoted by $\left.\mathcal{F}\right|_{L}$ ) is an algebra of functions on $L$, closed under conjugation and separating points. Since $L$ is a finite set (hence compact in the discrete topology), by the Stone-Weierstrass theorem, the algebra $\left.\mathcal{F}\right|_{L}$ is dense in the collection of functions on $L$, hence actually equal to the collection of all functions on the finite set $L$. Since $L$ is sequentially good for functions in $\left.\mathcal{F}\right|_{L}$, we see that $L$ is good and not a loop. This contradiction shows that $S$ is good. We have proved:

If $S$ is sequentially good for an algebra of functions on $S$ closed under conjugation and separating points of $S$, then $S$ is good for all functions on $S$, hence a good set.

Now assume that $m$ is a sequentially good probability measure on $X \times Y$. Let $\mathcal{C}$ be a countable collection of Borel sets which separates points of $X \times Y$, and is closed under finite unions and complements. Since $m$ is sequentially good, after deleting a set of measure zero, we can get a Borel set $S$ such that every $1_{A}, A \in \mathcal{C}$, is of the form

$$
1_{A}(x, y)=\lim _{n \rightarrow \infty}\left(u_{n}(x)+v_{n}(y)\right)
$$

for all $(x, y) \in S, u_{n}, v_{n}$ being Borel functions on $X$ and $Y$ respectively. (Here we have used the fact that every sequence converging in $L^{2}$ admits a subsequence converging a.e., and that if there are countably many sequences converging in $L^{2}$ then by a diagonal procedure we can find a single subsequence of integers over which each of the countably many sequences converges a.e.) The same continues to hold for the collection $\mathcal{F}$ of all finite linear combinations of $1_{A}, A \in \mathcal{C}$, and since $\mathcal{C}$ is closed under finite unions and complementation, $\mathcal{F}$ is an algebra of functions on $S$ which separates points and is closed under conjugation. We see that $S$ is a good set of full $m$-measure. We have proved:

4.4. TheOREM. If $m$ is sequentially good, then $m$ admits a Borel support $S$ which is good. 
In $[2$, Theorem 2.4] the following fact is proved:

4.5. TheOrem. If every $f \in L_{0}^{2}(X \times Y, m)$ (=functions in $L^{2}(X \times Y, m)$ with vanishing integral) can be expressed in the form

$$
f(x, y)=u(x)+v(y) \quad m \text {-a.e. }
$$

with $u, v$ square integrable and $\int u=\int v=\int u \bar{v}=0$, then $m$ is supported on a set of the form $G \cup H$, where $G$ is the graph of a Borel function $g$ defined on a Borel subset $A$ of $X$ and $H$ is the graph of a function $h$ defined on a Borel subset $B$ of $Y$ with $g(A) \cap H=G \cap h(B)=\emptyset$.

In the light of this and the discussions of the previous sections, it seems natural to conjecture that if $m$ is sequentially good, then there is a good Borel set which supports $m$ and which can be written as a union of two Borel graphs.

\section{Connection with dynamics}

5.1. Let $T_{1}$ be a Borel isomorphism from $X$ onto $Y$ and $T_{2}$ a Borel isomorphism from $Y$ onto $X$, where $X$ and $Y$ are standard Borel spaces, with $\sigma$-algebras $\mathcal{B}_{X}$ and $\mathcal{B}_{Y}$ respectively (isomorphic to the unit interval). Define the Borel automorphism $T$ of $\Omega=X \times Y$, equipped with the product $\sigma$-algebra $\mathcal{B}_{\Omega}=\mathcal{B}_{X} \otimes \mathcal{B}_{Y}$, by

$$
T(x, y)=\left(T_{2} y, T_{1} x\right), \quad(x, y) \in X \times Y .
$$

Let $S \subset \Omega$. Then a linked component of $S$ is mapped by $T$ onto a linked component of $T S$, and a uniquely linked component of $S$ is mapped onto a uniquely linked component of $T S$. If $T S=S$, then $T$ acts on the partition $\mathcal{P}$ of linked components.

5.2. Let $m$ be a sequentially good continuous probability measure on $\mathcal{B}_{\Omega}$, invariant under $T$. Let $S \subset \Omega$ be a good Borel set invariant under $T$ and supporting $m$. We assume that the partition $\mathcal{P}$ of $S$ into uniquely linked components admits a Borel cross-section, so that the quotient $\sigma$-algebra $\mathcal{Q}$ on $\mathcal{P}$ is countably generated. The transformation $T$ acting on $(\mathcal{P}, \mathcal{Q})$ is then a factor of $T$. Assume now that $T$ is prime. This means that either $\mathcal{Q}=$ $\mathcal{B}_{\Omega}(\bmod m)$, or $\mathcal{Q}=\{\Omega, \emptyset\}(\bmod m)$. If $\mathcal{Q}=\mathcal{B}_{\Omega}(\bmod m)$, then there is a Borel cross-section $C$ of $\mathcal{P}$ of measure one, so that $m$ is supported on the graph of a one-to-one function. We show that the other possibility, viz., $\mathcal{Q}=\{\Omega, \emptyset\}(\bmod m)$, cannot arise. For then $m$ is supported on a uniquely linked component, say $L$, of $S$. If $(a, b) \in L$, then, in the notation of Theorem 2.12, we have:

$$
L=G(a, b) \cup H(a, b), \quad G(a, b) \cap H(a, b)=\{(x, y)\}=\emptyset(\bmod m),
$$




$$
\begin{aligned}
& T G(a, b)=H(T(a, b))=H(x, y)(\bmod m), \\
& T H(a, b)=G(T(a, b))=G(x, y)(\bmod m),
\end{aligned}
$$

so that $G(a, b)$ generates a non-trivial $\sigma$ algebra which, modulo $m$, is invariant under $T$. Since $T$ is prime, we have a contradiction. Thus, if $T$ is prime, then $m$ is supported on the graph of a one-to-one function.

If one relaxes the primality condition on $T$ but requires only that $T^{2}$ is ergodic, then one can prove that $m$ is supported on a countable union of Borel graphs of one-to-one functions (the other conditions, namely that $m$ is continuous, sequentially good and that $\mathcal{P}$ admits a Borel cross-section still being in force). To this end let $C$ be a Borel cross-section of $\mathcal{P}$. Then $C$ is the graph of a one-to-one Borel function and so is each $T^{n} C$. If $m(C)>0$, then, since $T$ is ergodic, $m$ is supported on $\bigcup_{n=0}^{\infty} T^{n} C$, a countable union of graphs of one-to-one Borel functions. If $m(C)=0$, we show that $T^{2}$ is not ergodic. With notation of Theorem 2.12, let

$$
G=\bigcup_{(a, b) \in C} G(a, b), \quad H=\bigcup_{(a, b) \in C} H(a, b) .
$$

Then $m(H \cup G)=1, G \cap H=C$, and

$$
T G=\bigcup_{(a, b) \in C} T G(a, b)=\bigcup_{(a, b) \in C} H(T(a, b))=\bigcup_{(a, b) \in T C} H(a, b) .
$$

Similarly $T H=\bigcup_{(a, b) \in T C} G(a, b)$. We see that $T G \triangle H \subset C \cup T C$, and since $m(C)=0$, we see that $T G=H(\bmod m)$, while $m(G \cap H)=m(C)=0$. Thus $T^{2} G=G(\bmod m)$ with $m(G)=1 / 2$, contradicting the ergodicity of $T^{2}$. So $m(C)>0$. We have proved:

5.3. Theorem. Let $\Omega, m, T, \mathcal{P}$ be as above with $m$ continuous and sequentially good, while $\mathcal{P}$ admits a Borel cross-section. If $T$ is prime, then $m$ is supported on the graph of a one-to-one Borel function. The automorphisms $T^{2}, T_{1} \circ T_{2}$ and $T_{2} \circ T_{1}$ are then isomorphic. If we assume only that $T^{2}$ is ergodic, then $m$ is supported on a countable union of Borel graphs of one-to-one functions.

5.4. Returning to the notation of Section 1 we see that if the closed linear span of the process $X_{k}, k \in \mathbb{Z}$, is equal to $L^{2}(\Omega, \mu)$, then $\mu$ is supported on a good Borel subset $S$ of $\Omega_{A} \times \Omega_{B}$; in case $A=2 \mathbb{Z}$ and $B=2 \mathbb{Z}+1$, we can choose $S$ to be $T$-invariant. If the partition of $S$ into uniquely linked components admits a Borel cross-section and $T$ is prime, then $\mu$ is supported on the graph of a one-to-one function, so that the actions of $T^{2}$ on $\Omega, \Omega_{2 \mathbb{Z}}$, $\Omega_{2 \mathbb{Z}+1}$ are isomorphic. If we merely assume that $T^{2}$ is ergodic (and $P$ admits a Borel cross-section), then $\mu$ is supported on a countable union of graphs of one-to-one Borel functions on $\Omega_{2 \mathbb{Z}}$. It seems plausible that in Theorem 5.3 the partition $\mathcal{P}$ can always be chosen to admit a Borel cross-section. 
It is a pleasure to acknowledge some fruitful discussions with H. Sarbadhikari and S. M. Srivastava.

\section{REFERENCES}

[1] R. C. Cowsik, A. Kłopotowski and M. G. Nadkarni, When is $f(x, y)=u(x)+$ $v(y)$ ?, Proc. Indian Acad. Sci. (Math. Sci.) 109 (1999), 57-64.

[2] A. Kłopotowski and M. G. Nadkarni, On transformations with simple Lebesgue spectrum, ibid., 47-55.

[3] M. Laczkovich, Closed sets without measurable matchings, Proc. Amer. Math. Soc. 103 (1998), 894-896.

Institut Galilée

Université Paris XIII

93430 Villetaneuse Cedex, France

E-mail: klopot@math.univ-paris13.fr
Department of Mathematics University of Mumbai

Kalina, Mumbai, India 400098

E-mail:nadkarni@math.mu.ac.in 